\title{
Perceptions Des Nuisances Environnementales Liées Aux Déchets Solides Ménagers Dans Les Centres Urbains Du Nord-Bénin : Cas Des Villes De Parakou, Djougou, Kandi Et Malanville
}

\author{
Babio Sanni \\ Baloubi Makodjami David \\ Laboratoire d'Etudes des Dynamiques Urbaine et Régionale \\ /DGAT/FLASH/UAC ; Cotonou - BENIN \\ Houssou Sègbè Christophe \\ Laboratoire Pierre PAGNEY ' 'Climat, Eau, Ecosystèmes et \\ Développement”' (LACEEDE/UAC), Abomey-Calavi, BENIN
}

doi: 10.19044/esj.2016.v12n23p349 URL:http://dx.doi.org/10.19044/esj.2016.v12n23p349

\begin{abstract}
The cities of Parakou, Djougou, Kandi and Malanville in the north of Benin have difficulty in eliminating dispose of waste produced by households in ecological way. This research was initiated to analyze the attitudes and understanding of environmental problems related to poor management of DSM by people of north Benin. To achieve this objective, a survey was conducted on the perception of environmental damage related to waste with 585 heads of households from August 2014 to March 2015. The city of residence, gender, age, marital status, level education, income, socio professional category are characteristics that were selected for the statistical analysis Khi2. Results showed $100 \%$ a significant statistical link between environmental pollution and city of residence $(\chi 2=153.135$; DF $=12$; Pvalue $=0.000<0.05)$. No statistically significant association was found between perceived environmental nuisances and sex $(\chi 2=2.035$; $\mathrm{DF}=4$; PValue $=0.729>0.05) ;$ age $(\chi 2=19.189, \mathrm{DF}=16 ; \mathrm{P}-$ Value $=0.259>0.05)$; marital status $(\chi 2=20.809$, DF $=12$; P-Value $=0.053>0.05$ and income of respondents $(\chi 2=32.273, \mathrm{DF}=32$; P-Value $=0.453>0.05)$. However in Djougou, a highly significant associative link was observed between respondents with incomes ranging from 100,001 to 150,000 and the air pollution $(\chi 2=11.96, \mathrm{DF}=32$; $\mathrm{P}$-value $=0.000<0.05)$. It is the same for the pollution of rivers in this income bracket in Parakou $(\chi 2=5.85$; DF $=24$; Pvalue $=0.004<0.05)$ and Kandi $(\chi 2=604 ; \mathrm{DF}=10 ; \mathrm{P}$-value $=0.000<$. $0.05)$. Moreover, the level of education $(\chi 2=14.728, \mathrm{DF}=12$; $\mathrm{P}-$ Value $=$
\end{abstract}


$0.257>0.05)$ and occupation of the individuals interviewed $(\chi 2=59.060, \mathrm{DF}$ $=40 ;$ P-Value $=0.026<0.05$ ) determine their perceptions of environmental damage related to DSM. In total, city of residence, education level and occupation of individuals are the most, significant determiners in the perception of environmental damage related to the solid household waste.

Keywords: Perception - nuisance - Environmental - household wastes

\section{Résumé}

Les villes de Parakou, Djougou, Kandi et Malanville au nord-Bénin éprouvent des difficultés à éliminer écologiquement les déchets produits par les ménages. C'est pour analyser les attitudes et la compréhension des problèmes environnementaux liés à la mauvaise gestion des DSM par les populations au nord-Bénin que cette recherche a été initiée. Pour y parvenir, une enquête a été réalisée sur la perception des nuisances environnementales liées aux déchets auprès de 585 chefs de ménages d'août 2014 à mars 2015. La ville de résidence, le sexe, l’âge, le statut matrimonial, le niveau d'instruction, le revenu, la catégorie socio-professionnelle et le type de quartiers ont été les attributs retenus pour l'analyse statistique Khi2. Les résultats ont révélé à $100 \%$ un lien statistique très significatif entre nuisances environnementales et la ville de résidence $(\chi 2=153,135$; DF $=12$; P-Value $=0,000<0,05)$. Aucun lien statistiquement significatif n'a été décelé entre perception des nuisances environnementales et le sexe $(\chi 2=2,035$; $\mathrm{DF}=4$; P-Value $=0,729>0,05)$; l'âge $(\chi 2=19,189 ; \mathrm{DF}=16 ; \mathrm{P}-$ Value $=0,259>0,05)$ ; le statut matrimonial $(\chi 2=20,809 ; \mathrm{DF}=12$; P-Value $=0,053>0,05$ et le revenu des répondants $(\chi 2=32,273 ; \mathrm{DF}=32 ; \mathrm{P}-$ Value $=0,453>0,05)$. Toutefois, à Djougou, un lien associatif hautement significatif a été observé entre les personnes interrogées à revenu variant de 100001 à 150000 et la pollution de l'air $(\chi 2=11,96 ; \mathrm{DF}=32$; P-Value $=0,000<0,05)$. Il en est de même pour la pollution des cours d'eau dans cette tranche de revenu à Parakou $(\chi 2=5,85 ; \mathrm{DF}=24$; P-Value $=0,004<0,05)$ et à Kandi $(\chi 2=6,04$; $\mathrm{DF}=10$; P-Value $=0,000<0,05)$. Par ailleurs, le niveau d'instruction $(\chi 2=$ 14,$728 ; \mathrm{DF}=12$; P-Value $=0,257>0,05$ ) et la profession des individus interrogés $(\chi 2=59,060 ; \mathrm{DF}=40 ; \mathrm{P}-$ Value $=0,026<0,05)$ déterminent leurs perceptions des nuisances environnementales liées aux DSM. Au total, la ville de résidence, le niveau d'instruction et la profession des individus sont les déterminants les plus significatifs dans la perception des nuisances environnementales liées aux déchets solides ménagers.

Mots clés : Déchets-ménagers -perception - nuisance - environnementale 


\section{Introduction}

La gestion des déchets solides ménages (DSM), constitue un des principaux défis auquel sont confrontées les villes, du Nord comme celles du Sud (Wang \& al. 2014). L'ampleur de ce problème se ressent avec une extrême acuité dans les villes des pays en développement où les dynamiques urbaines sont mal maitrisées (Raab et Gobin, 2010). En Afrique, les difficultés de gestion des déchets ont atteint des proportions telles que les mesures prises par les différents niveaux d'administration et les spécialistes se sont révélées infructueuses (Onibokun, 2002). Au Bénin, la situation est caractérisée par une incapacité notoire des collectivités locales à assurer l'élimination adéquate des DSM (Eyébiyi, 2010). En effet, la plupart des villes du Bénin, sont confrontées à une forte croissance de la population (Guegant, 2011) et à une évolution des modes de consommation qui se traduisent par une augmentation des volumes et de la nature des déchets (Gbinlo, 2010). Dans le même temps, on note une insuffisance de ressources humaines, techniques et financières. Du coup, dans presque toutes les villes comme celles de Parakou, Djougou, Kandi et Malanville, les ruelles, les basfonds, les parcelles non bâties sont transformés en dépotoirs de déchets solides ménagers au gré des comportements peu civiques des populations. Ces dépotoirs sauvages dégagent souvent d’odeurs nauséabondes pendant la fermentation des déchets. Cette situation qui engendre des nuisances importantes pour les habitants, a des conséquences néfastes sur la santé des populations, sur l'environnement et sur les ressources. L'objectif de cette recherche est d'analyser les attitudes et la compréhension des problèmes environnementaux liés à la mauvaise gestion des DSM par les populations au nord-Bénin en vue d'une meilleure gestion de l'hygiène publique.

\section{Méthodes}

\section{Milieu d'étude}

Les villes de Parakou, Djougou, Kandi et Malanville font partie des principaux centres urbains du nord-Bénin (figure 1). 


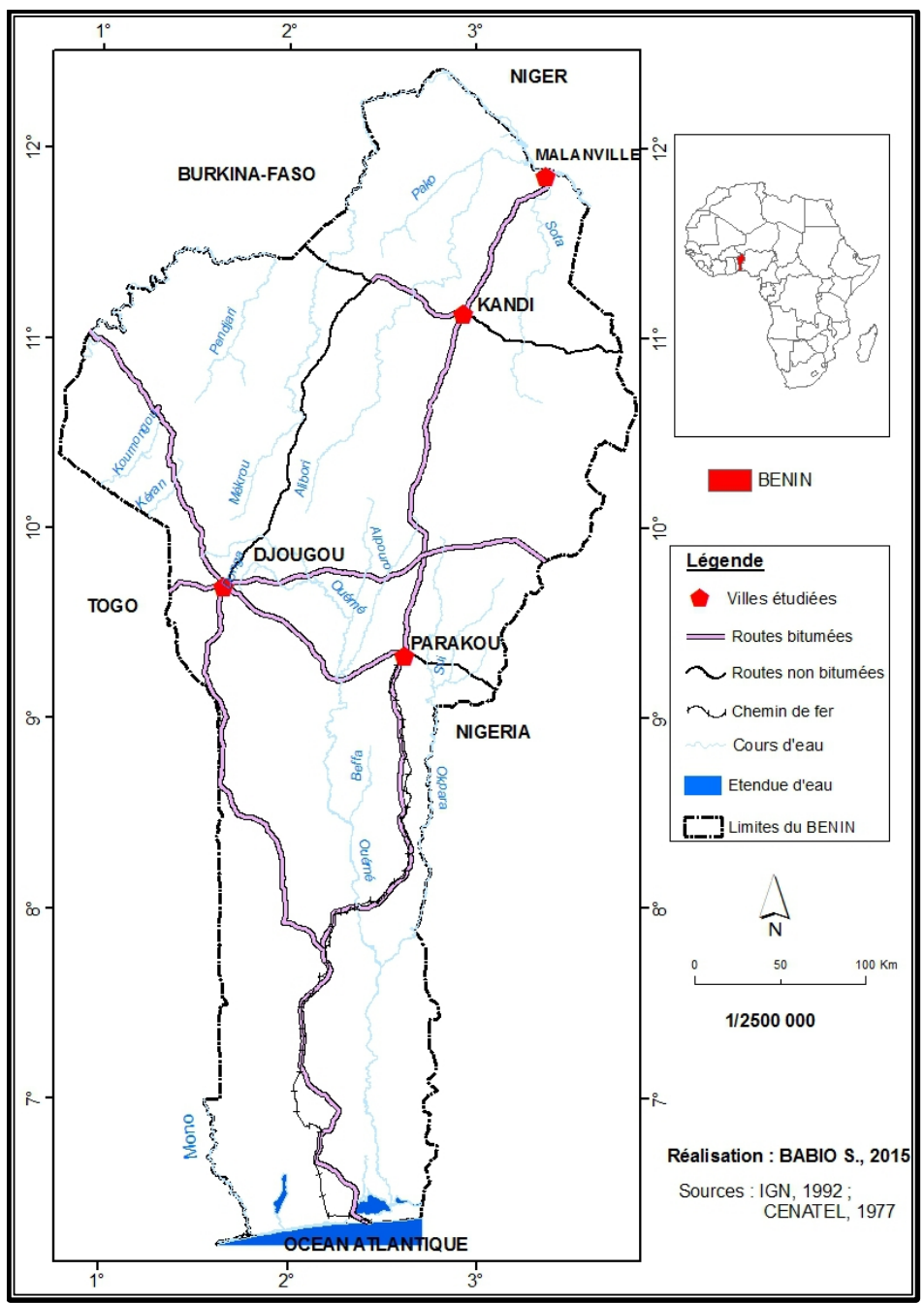

Figure 1 : situation géographique des villes de Parakou, Djougou, Kandi et Malanville au Bénin

\section{La ville de Parakou}

Située entre $9^{\circ} 18^{\prime} 15^{\prime \prime}$ et $9^{\circ} 24^{\prime} 35^{\prime \prime} \mathrm{N}$ et entre $2^{\circ} 34^{\prime}$ 'et $2^{\circ} 40^{\prime}$, la ville de Parakou (figure 1) est le chef-lieu des départements du Borgou/Alibori. Elle constitue un important centre administratif, bancaire, industriel et universitaire du nord-Bénin. La ville se classe troisième dans la hiérarchie des principales villes du Bénin après Cotonou et Porto-Novo. Sa population en 2013 est de 255478 habitants (INSAE, 2015), d'où l'importance des rejets de déchets que les autorités locales ont du mal à éliminer. La ville jouit d'un climat tropical humide de type continental, dominé par une saison des pluies et une saison sèche. Les températures moyennes mensuelles oscillent entre 25 et $31^{\circ} \mathrm{C}$. 


\section{La ville de Djougou}

Djougou est une ville située dans la partie Nord-Ouest du Bénin (figure 1) entre 9 $41^{\prime} 10^{\prime \prime}$ et $9^{\circ} 43^{\prime} 35^{\prime \prime} \mathrm{N}$ et entre $1^{\circ} 38^{\prime} 40^{\prime \prime}$ et $1^{\circ} 41^{\prime} 20^{\prime \prime} \mathrm{E}$. C'est est une ville carrefour et de transit située sur les principaux axes routiers menant vers le Togo, le Burkina-Faso et le Niger. Selon l'INSAE, (2015) sa population en 2013 est de 94773 habitants. La ville bénéficie d'un climat soudano-guinéen marqué par des précipitations moyennes annuelles qui varient entre 1000 et $1500 \mathrm{~mm}$. Cette importante pluviométrie est un facteur favorable à la décomposition des déchets organiques et par conséquent à la multiplication des vecteurs.

\section{La ville de Kandi}

Elle est une ville du nord-Bénin (figure 1) située entre 2०54'57'” et $2^{\circ} 57^{\prime} 4^{\prime \prime}$ E et entre $11^{\circ} 6^{\prime} 56^{\prime \prime}$ et $11^{\circ} 8^{\prime} 533^{\prime \prime}$ N. Son climat est du type soudanien caractérisé par deux saisons (pluvieuse et sèche) bien tranchées avec des hauteurs annuelles de pluies qui varient considérablement entre 700 et $1150 \mathrm{~mm}$. En 2013, la population de la ville était de 56037 habitants (INSAE, 2015).

\section{La ville de Malanville}

La ville de Malanville est située à l'extrême Nord du Bénin (figure 1)

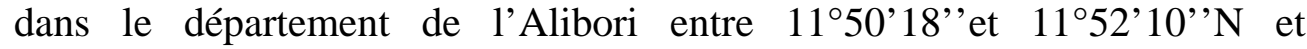
$3^{\circ} 22^{\prime} 20^{\prime \prime}$ et $3^{\circ} 2418^{\prime \prime} \mathrm{E}$. Cette ville carrefour constitue un important pôle économique et abrite le deuxième marché international du Bénin après Dantokpa à Cotonou. Sa population en 2013 est de 64639 habitants (INSAE, 2015). Son climat est de type soudano-sahélien avec une saison sèche de novembre à avril et une saison pluvieuse de mai à octobre. Les hauteurs moyennes annuelles de pluies oscillent autour de $750 \mathrm{~mm}$. Les températures sont très élevées avec des amplitudes thermiques pouvant atteindre $25^{\circ} \mathrm{C}$. Au plan pédologique, les sols sont de types sablo-argilo et ferrugineux et alluviaux sablo-limoneux très perméables à l'infiltration du lixiviat.

\section{Collecte des données}

\section{Echantillonnage}

Afin d'analyser la perception des populations sur les nuisances environnementales liées aux déchets solides ménagers (DSM) dans les villes de Parakou, de Djougou, de Kandi et de Malanville, une trame de questionnaire a été adressée à un échantillon de $5 \%$ des ménages dans les principaux quartiers de ces villes. Ainsi, 585 ménages ont été interrogés dont 230 à Parakou, 155 à Djougou, 125 à Malanville et 75 à Kandi. 


\section{Définition des variables à analyser}

Une meilleure gestion des DSM dans les villes ne saurait être possible sans une connaissance fine de la perception des populations des risques liés sur à ces déchets. Car, «chaque individu développe un lien particulier avec le territoire concerné par le risque et le perçoit de manière personnelle, allant de l'acceptation, à son déni, en passant par l'indifférence liée à la non connaissance des risques» (Trémolières, 2005 cité par Kientga, 2008). Pour les besoins de cette recherche, la perception est définie comme un processus au cours duquel un individu utilise un ou plusieurs de ses sens pour appréhender l'information qui lui parvient et interpréter un ou plusieurs stimuli auxquels il est soumis (Lehu, 2004). Pour percevoir il faut donc soit voir, soit entendre, soit toucher, soit sentir, soit goûter, soit un mélange de ces cinq activités. Plusieurs facteurs sont souvent utilisés pour analyser la perception. Il s'agit des :

attributs personnels tels que l'âge, le sexe, la catégorie socioprofessionnelle et le niveau d'instruction (Vining et al., 1992; Samdahl et Robertson, 1989; Neuman, 1986; Canteret al., 1992) ;

a attributs familiaux comme le statut matrimonial et le revenu (Hamilton, 1985; Renn et al., 1992; Vining et al., 19 92) ;

attributs géographiques (ville de résidence et types de quartier).

Les nuisances environnementales retenues (tableau 1) ont été classées en cinq groupes et codées afin de faciliter le traitement statistique.

Tableau 1 : nuisances environnementales citées par les ménages et leurs codes d'identification

\begin{tabular}{ll}
\hline Types de pollutions & Codes \\
\hline Pollution de l'air & P.air \\
Pollution des sols & P.sols \\
Pollution des eaux & P.eaux \\
Dénaturation de l'environnement urbain & D.env.urb. \\
Pollution et dénaturation de l'environnement urbain & P.air+ D.env.urb. \\
\hline
\end{tabular}

Source : enquête de terrain, 2015

\section{Traitement et analyse des données}

Toutes les données collectées ont été codées et traités avec le logiciel IBM Spss statistics 21 et les résultats présentés sous formes de tableaux de contingence ou de graphiques. Ces résultats ont par la suite été analysés par le test de khi2 (Barnier, 2013) à partir du logiciel Minitab pour apprécier l'existence ou non de relation statistiquement significative entre les nuisances environnementales déclarées et les attributs socio-économiques retenus pour cette analyse. Pour que l'on puisse affirmer qu'il existe une relation entre attributs de chefs de ménages et nuisances environnementales, il faut que la p-value soit inférieure à 0,05. Il s'agit en fait d'un seuil de risque de $5 \%$ (ou niveau de confiance de 95\%). Ainsi, si la «p-value » est 
inférieure ou égale à 0,05 , on peut affirmer avec moins de $5 \%$ de chances de se tromper qu'il existe une relation statistiquement les variables étudiées. De même, si «p-value»< 0,01, la relation est hautement significative ; «pvalue»<0,001 la relation est dite très hautement significative.

\section{Résultats}

\section{Perception des nuisances environnementales selon la ville de résidence}

La sensibilité aux nuisances environnementales liées aux déchets solides ménagers des populations varie d'une ville à une autre. En effet, les résultats des enquêtes réalisées auprès de 585 chefs de ménages dans les villes de Parakou, Djougou, Kandi et Malanville ont révélé, à 100\% un lien statistique très significatif entre nuisances environnementales et la ville de résidence $(\chi 2=153,135$; DF $=12 ; \mathrm{P}-$ Value $=0,000<0,05)$. Par exemple à Kandi (64\%) et à Djougou (52,26\%), les populations ont signalé plus la pollution de l'air qu'à Parakou (25,65\%) et à Malanville (20\%). Dans le même temps à Malanville (55,2\%) et à Parakou (49,13\%), la pollution des sols est une préoccupation essentielle pour les ménages (figure 2). Dans l'ensemble des villes étudiées, une majorité relative (38,29\%) des répondants a signalé la pollution des sols comme nuisance environnementale majeure dans leur localité. Cela traduit un agacement des populations face à l'insalubrité presque chronique de leur localité que les différents programmes de "'ville propre' n’ont pas pu réduire.

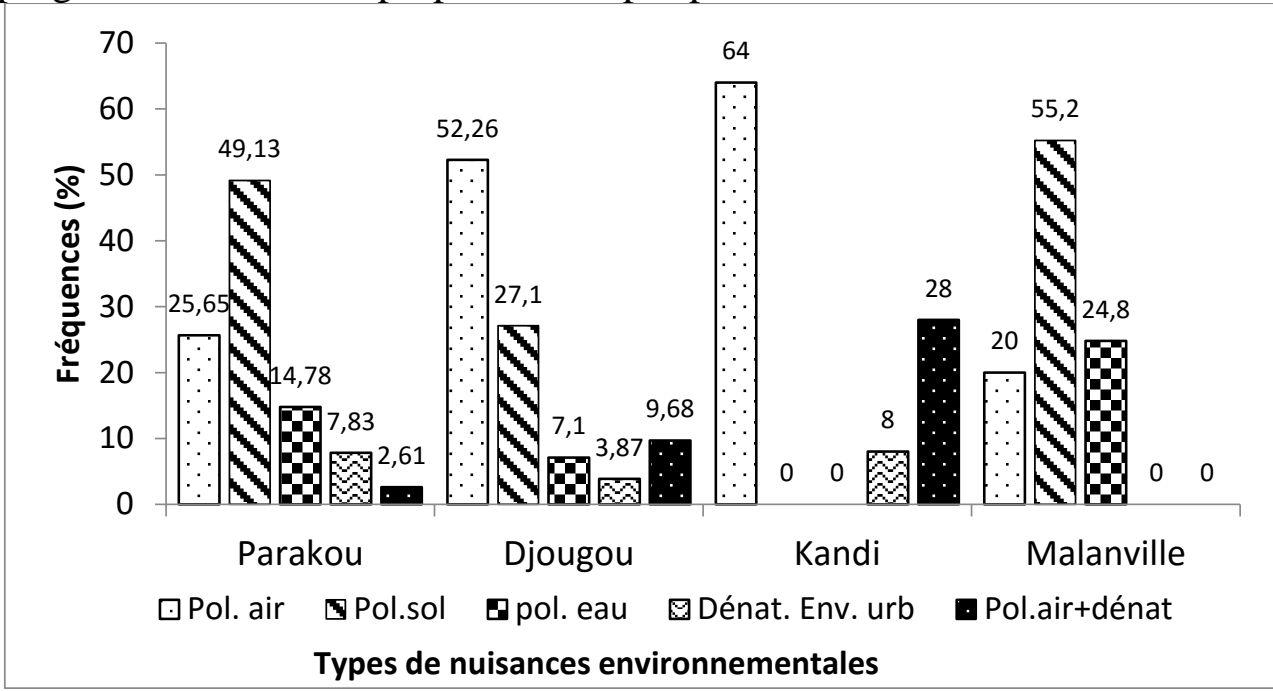

Figure 2: perceptions des nuisances environnementales liées aux déchets solides ménagers dans les villes étudiées

\section{Perception des nuisances environnementales selon le sexe}

La sensibilité aux nuisances environnementales dans les villes de Parakou, de Djougou, de Kandi et de Malanville n’est pas fonction du sexe 
des personnes interrogées $(\chi 2=2,035 ; \mathrm{DF}=4$; $\mathrm{P}$-Value $=0,729>0,05)$. Toutefois, les femmes plus que les hommes, ont signalé la pollution des sols à 39,49\% (tableau 2) alors que les hommes s’attardent davantage à la pollution de l'air (36,45\%).

Tableau 2 : perceptions des nuisances environnementales selon le sexe

\begin{tabular}{|c|c|c|c|c|c|}
\hline \multirow{2}{*}{ Types de pollution } & \multicolumn{2}{|c|}{ Masculin } & \multicolumn{2}{|c|}{ Féminin } & \multirow{2}{*}{ Total } \\
\hline & $\mathrm{N}$ & $\%$ & $\mathrm{~N}$ & $\%$ & \\
\hline Pollution de l'air & 156 & 36,45 & 57 & 36,31 & 213 \\
\hline Pollution des sols & 162 & 37,85 & 62 & 39,49 & 224 \\
\hline Pollution des eaux & 56 & 13,08 & 20 & 12,74 & 76 \\
\hline $\begin{array}{l}\text { Dénaturation } \\
\text { l'environnement urbain }\end{array}$ & 25 & 5,84 & 5 & 3,18 & 30 \\
\hline $\begin{array}{l}\text { Odeur et dénaturation de } \\
\text { l'environnement }\end{array}$ & 29 & 6,78 & 13 & 8,28 & 42 \\
\hline Total & 428 & 100 & 157 & 100 & 585 \\
\hline
\end{tabular}

Source : enquêtes de terrain, 2015

Cette tendance est aussi observée dans la ville de Djougou où les hommes (55,05\%) sont plus sensibles à la pollution de l'air que les femmes $(45,65)$. En effet, dans les sociétés africaines en général et dans le contexte béninois en particulier, la propreté des maisons est un rôle dévolu aux femmes. C'est à juste titre qu'elles sont plus sensibles à la pollution des sols (Figure 3).

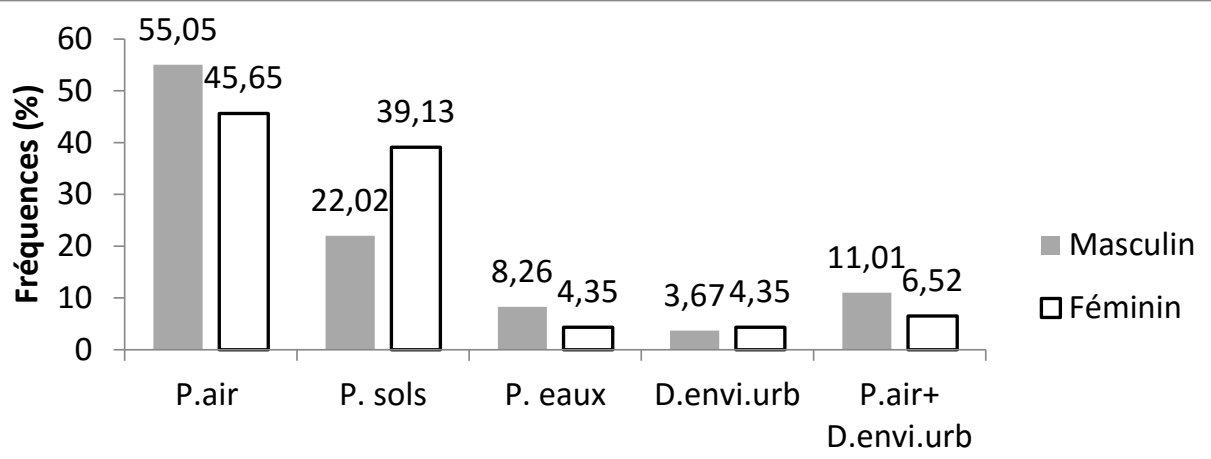

Types de nuisance environnementales

Figure 3 : Perceptions des nuisances environnementales liées aux déchets ménagers selon le sexe dans la ville de Djougou

\section{Perception des nuisances environnementales selon l’âge}

Les enquêtes effectuées sur les quatre villes étudiées n’ont révélé aucun lien statistique significatif entre nuisances environnementales liées aux déchets et l'âge des populations interrogées $(\chi 2=19,189$; $\mathrm{DF}=16$; P-Value = $0,259>0,05)$. Cependant, chez les répondants actifs : 21 à 30 ans (41,94\%); 31 à 40 ans (39,55\%) et 41 à 50 ans (35,51\%), le degré de sensibilité à la 
pollution de l'air (figure 4) est plus élevé que chez les personnes de 61 à plus de 70 ans (29,\%). En effet, à plus de 61 ans, les hommes sont pour la plupart moins actifs, se déplaçant peu et donc moins exposés à la pollution atmosphérique.

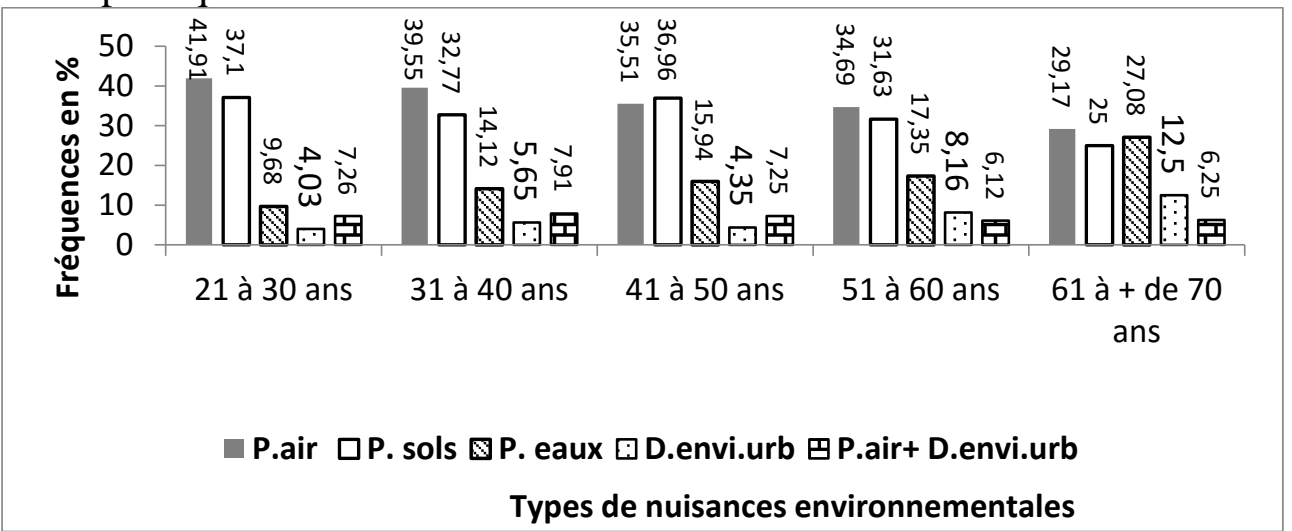

Figure 4: perceptions des nuisances environnementales liées aux déchets ménagers selon l'âge

Perception des nuisances environnementales selon le statut matrimonial

Dans l'ensemble des villes étudiées, le statut matrimonial des personnes interrogées n'influence pas leur perception des nuisances environnementales liées aux DSM $(\chi 2=20,809 ; \mathrm{DF}=12 ; \mathrm{P}$-Value $=$ $0,053>0,05)$. Toutefois, les répondants célibataires sont plus sensibles (52,94\%) à la pollution des sols (figure 5) que les autres. En effet, le célibataire qui vit seul est sensé produire et rejeter moins de déchets et ainsi plus sensible à pollution des sols engendrée par des ménages où habitent plus d'individus. Car plus il existe de consommateurs, plus il y a de risque de rejet et donc de pollution.

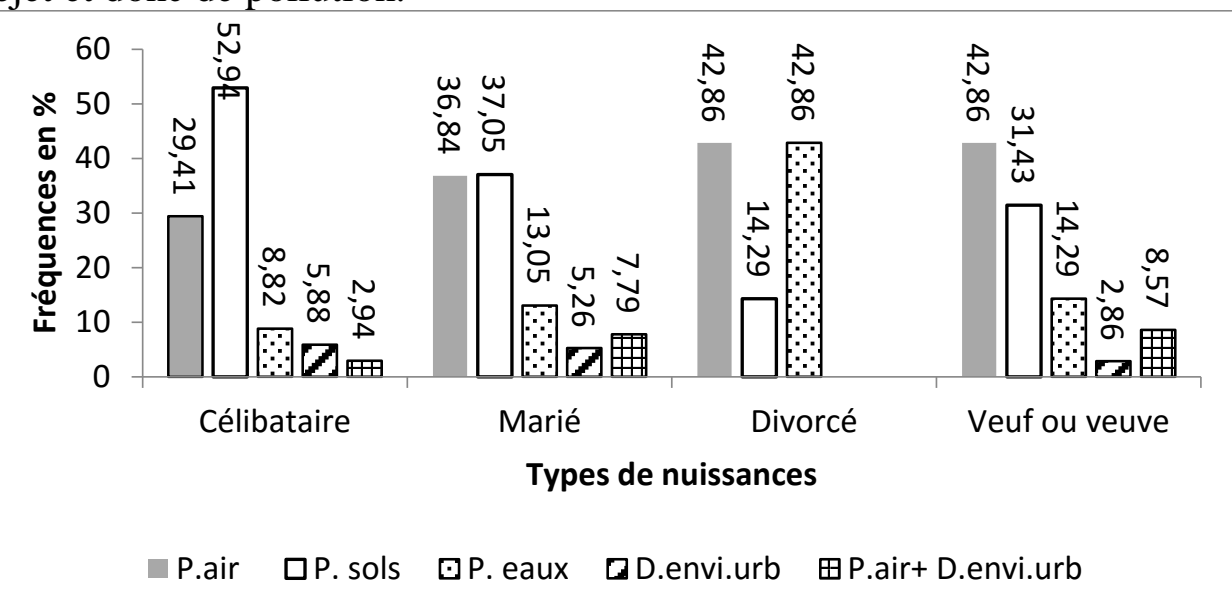

Figure 5 : perceptions des nuisances environnementales liées aux déchets ménagers selon le statut matrimonial 


\section{Perception des nuisances environnementales selon le niveau d'instruction}

Aucun lien statistiquement significatif n’a été enregistré entre la perception des nuisances environnementales et le niveau d'instruction des populations interrogées $(\chi 2=14,728 ; \mathrm{DF}=12$; P-Value $=0,257>0,05)$. Toutefois la sensibilité à la pollution de l'air liée aux déchets est plus élevée chez les répondants non instruits : 38,40\% contre 31,69 \% et 31,11\% pour respectivement les interrogés des niveaux secondaire et supérieur. En effet, dans la plupart des centres urbains étudiés, les non instruits sont rencontrés majoritairement dans les quartiers populaires et périphériques. Hors dans ces quartiers, le système de pré collecte d'ordures est quasiment absent. Parfois, les quartiers périphériques servent d'exutoire aux autres quartiers. Car, les déchets collectés de l'intérieur sont acheminés vers la périphérie, polluant davantage l'atmosphère aux riverains de ces quartiers. La sensibilité à la pollution des cours d'eau augmente avec le niveau d'instruction : 10,27\% chez les non instruits ; $11,27 \%$ pour le niveau du primaire ; $16,30 \%$ pour le secondaire et 24,44 pour le niveau supérieur (figure 6). En effet, avec un niveau d'instruction élevé, l'individu est plus informé sur les maladies hydriques. C'est pourquoi, les répondants instruits sont plus sensibles à la pollution de l'eau que les non instruits.

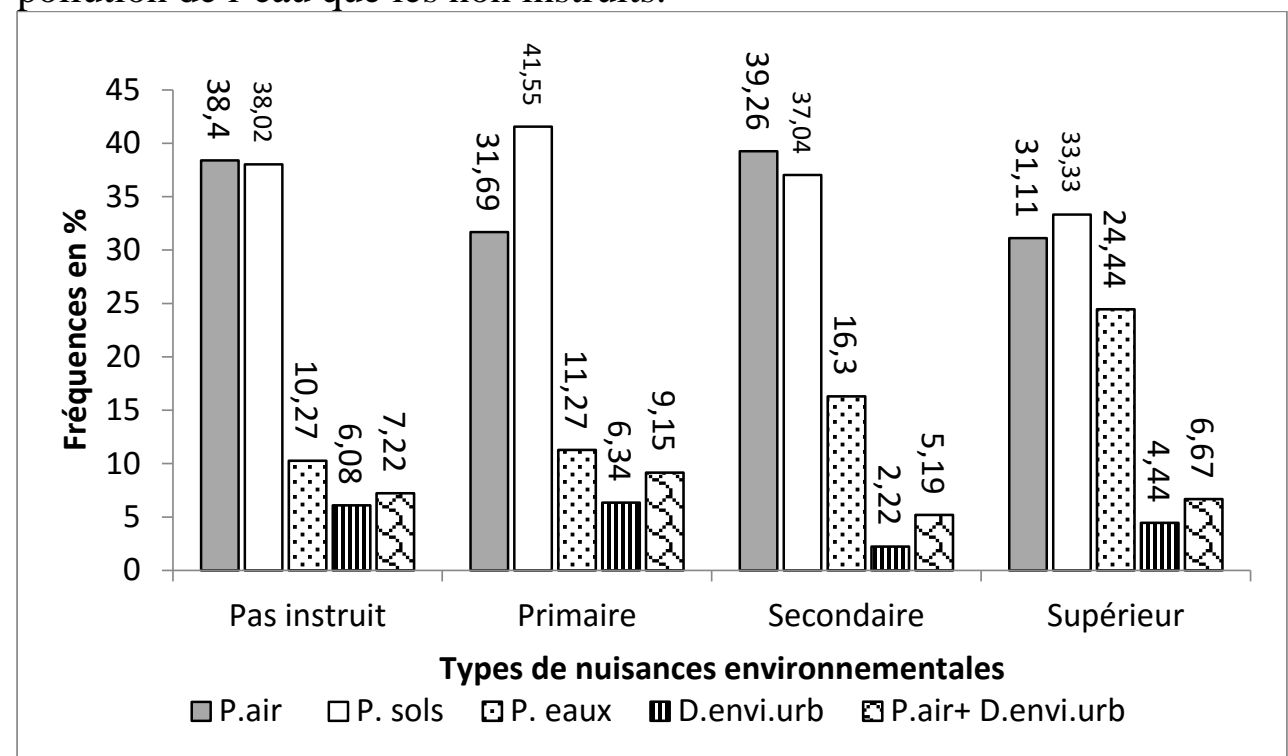

Figure 6 : perceptions des nuisances environnementales liées aux déchets ménagers selon les niveaux d'instruction 


\section{Perception des nuisances environnementales selon la catégorie socio- professionnelle}

La perception des nuisances environnementales liée aux DSM dépend en grande partie de la catégorie socio-professionnelle des chefs de ménages interrogés. En effet, un lien associatif a été observé entre perception des nuisances environnementales et la profession des individus interrogés $(\chi 2=$ 59,060; $\mathrm{DF}=40 ; \mathrm{P}-$ Value $=0,026<0,05)$. Le déplacement régulier de certains répondants en raison de leurs activités professionnelles ou à la recherche d'emploi les expose aux polluants atmosphériques. Par conséquent, ces derniers sont plus sensibles à la pollution de l'air. Ce sont les cas des transporteurs (51,28\%,), des chômeurs (41,67\%), des commerçants (40\%), des manœuvres (36,67\%) et des cadres de l'administration (33,33\%). Par ailleurs, la pêche étant une activité liée à l'eau, la pollution de cette dernière priverait les pêcheurs des ressources halieutiques importantes. C'est pourquoi, ils sont à 66,67\% (tableau 3) plus sensibles à cette forme de pollution que les transporteurs par exemple (7,69\%).

Tableau 3 : perceptions des nuisances environnementales liées aux déchets ménagers selon la catégorie socio-professionnelle

\begin{tabular}{|c|c|c|c|c|c|c|c|c|c|c|c|}
\hline \multirow{2}{*}{$\begin{array}{l}\text { Catégories socio } \\
\text { professionnelles }\end{array}$} & \multicolumn{2}{|c|}{ P.air } & \multicolumn{2}{|c|}{ P.sols } & \multicolumn{2}{|c|}{ P.eaux } & \multicolumn{2}{|c|}{ D.env.urb. } & \multicolumn{2}{|c|}{ P.air+ D.env.urb } & \multirow[t]{2}{*}{ Tota } \\
\hline & $\mathrm{N}$ & $\%$ & $\mathrm{~N}$ & $\%$ & $\mathrm{~N}$ & $\%$ & $\mathrm{~N}$ & $\%$ & $\mathrm{~N}$ & $\%$ & \\
\hline $\begin{array}{c}\text { Cadre de } \\
\text { l'administration }\end{array}$ & 25 & 33,33 & 31 & 41,33 & 12 & 16,00 & 3 & 4,00 & 4 & 5,33 & 75 \\
\hline Ouvrier qualifié & 17 & 29,82 & 18 & 31,58 & 14 & 24,56 & 5 & 8,77 & 3 & 5,26 & 57 \\
\hline Manœuvre & 11 & 36,67 & 8 & 26,67 & 7 & 23,33 & 3 & 10,00 & 1 & 3,33 & 30 \\
\hline Ménagère & 20 & 27,40 & 22 & 30,14 & 21 & 28,77 & 4 & 5,48 & 6 & 8,22 & 73 \\
\hline Producteur & 28 & 32,56 & 28 & 32,56 & 17 & 19,77 & 3 & 3,49 & 10 & 11,63 & 86 \\
\hline Pêcheur & 0 & 0,00 & 0 & 0,00 & 2 & 66,67 & 0 & 0,00 & 1 & 33,33 & 3 \\
\hline Commerçant & 54 & 40,00 & 58 & 42,96 & 12 & 8,89 & 5 & 3,70 & 6 & 4,44 & 135 \\
\hline Transporteur & 20 & 51,28 & 8 & 20,51 & 3 & 7,69 & 2 & 5,13 & 6 & 15,38 & 39 \\
\hline Artisan & 20 & 27,40 & 23 & 31,51 & 19 & 26,03 & 8 & 10,96 & 3 & 4,11 & 73 \\
\hline Chômeur & 5 & 41,67 & 2 & 16,67 & 1 & 8,33 & 2 & 16,67 & 2 & 16,67 & 12 \\
\hline Chef religieux & 2 & 100,00 & 0 & 0,00 & 0 & 0,00 & 0 & 0,00 & 0 & 0,00 & 2 \\
\hline Total & 213 & 36,41 & 224 & 38,29 & 76 & 12,99 & 30 & 5,13 & 42 & 7,18 & 585 \\
\hline
\end{tabular}

Source : enquêtes de terrain, 2015

\section{Perception des nuisances environnementales selon le niveau de revenu}

Aucun lien statistique significatif n’a été observé entre perception des nuisances environnementales liées aux DSM dans les villes étudiées dans leur ensemble et le niveau de revenu des personnes interrogées $(\chi 2=32,273$; $\mathrm{DF}=32$; P-Value $=0,453>0,05)$.

Ces résultats témoignent du fait contrairement à ce qu'on pouvait imaginer, les pauvres (faible revenu) sont aussi conscients des menaces sur leurs milieux de vie et par conséquent leurs perceptions des nuisances environnementales ne sont pas trop éloignées de celles des mieux nantis (tableau 4). Toutefois, les villes prises individuellement montrent des liens significatifs entre le revenu des personnes interrogées et la perception des 
nuisances environnementales. Ainsi, à Djougou, un lien associatif hautement significative a été observée entre les personnes interrogées à revenu variant de 100001 à 150000 et la pollution de de l'air $(\chi 2=11,96$; DF = 32 ; PValue $=0,000<0,05)$. Des liens ont été aussi observés entre cette tranche de revenu et la pollution des cours d'eau à Parakou $(\chi 2=5,85$; DF $=24$; P-Value $=0,004<0,05)$ et à Kandi $(\chi 2=6,04 ; \mathrm{DF}=10$; P-Value $=0,000<0,05)$. Par ces résultats on comprend que les nuisances environnementales loin de toutes considérations du niveau de revenu, sont liées à un espace géographique qui peut être un quartier ou une ville.

Tableau 4 : Perception des nuisances environnementales liées aux déchets ménagers selon le niveau de revenu

\begin{tabular}{|c|c|c|c|c|c|c|c|c|c|c|c|}
\hline \multirow{2}{*}{$\begin{array}{l}\text { Revenus } \\
\text { ménage }\end{array}$} & \multicolumn{2}{|c|}{ P.air } & \multicolumn{2}{|c|}{ P.sols } & \multicolumn{2}{|c|}{ P.eaux } & \multicolumn{2}{|c|}{ D.env.urb. } & \multicolumn{2}{|c|}{ P.air+D.env.urb } & \multirow{2}{*}{ Total } \\
\hline & $\mathrm{N}$ & $\%$ & $\mathrm{~N}$ & $\%$ & $\bar{N}$ & $\%$ & $\mathrm{~N}$ & $\%$ & $n$ & $\%$ & \\
\hline $\begin{array}{c}\text { Moins de } \\
30000\end{array}$ & 39 & 45,35 & 27 & 31,40 & 5 & 5,81 & 9 & 10,47 & 6 & 6,98 & 86 \\
\hline $\begin{array}{c}30000 \text { à } \\
500000\end{array}$ & 36 & 35,29 & 32 & 31,37 & 15 & 14,71 & 6 & 5,88 & 13 & 12,75 & 102 \\
\hline $\begin{array}{c}50001 \text { à } \\
75000\end{array}$ & 35 & 45,45 & 25 & 32,47 & 7 & 9,09 & 3 & 3,90 & 7 & 9,09 & 77 \\
\hline $\begin{array}{l}75001 \text { à } \\
100000\end{array}$ & 28 & 48,28 & 11 & 18,97 & 9 & 15,52 & 3 & 5,17 & 7 & 12,07 & 58 \\
\hline $\begin{array}{c}100001 \text { à } \\
150000\end{array}$ & 10 & 38,46 & 7 & 26,92 & 5 & 19,23 & 2 & 7,69 & 2 & 7,69 & 26 \\
\hline $\begin{array}{c}150001 \text { à } \\
200000\end{array}$ & 5 & 25 & 6 & 30 & 8 & 40 & 1 & 5 & 0 & 0 & 20 \\
\hline $\begin{array}{c}200001 \text { à } \\
300000\end{array}$ & 3 & 60 & 0 & 0 & 1 & 20 & 0 & 0 & 1 & 20 & 5 \\
\hline $\begin{array}{c}300001 \text { à } \\
400000\end{array}$ & 0 & 0 & 1 & 100 & 0 & 0 & 0 & 0 & 0 & 0 & 1 \\
\hline $\begin{array}{l}\text { Plus de } \\
500000\end{array}$ & 2 & 100 & 0 & 0 & 0 & 0 & 0 & 0 & 0 & 0 & 2 \\
\hline $\begin{array}{l}\text { Pas de } \\
\text { réponse }\end{array}$ & 55 & 26,44 & 115 & 55,29 & 26 & 12,50 & 6 & 2,88 & 6 & 2,88 & 208 \\
\hline Total & 213 & 36,41 & 224 & 38,29 & 76 & 12,99 & 30 & 5,13 & 42 & 7,18 & 585 \\
\hline
\end{tabular}

Source : enquêtes de terrain, 2015

Perception des nuisances environnementales selon le type de quartiers

Statistiquement, aucun lien significatif n’a été observé entre perception des nuisances environnementales liées aux DSM et les types de quartiers habités par les personnes interrogées $(\chi 2=11,775$; DF $=8$; P-Value $=0,162>0,05)$. Toutefois la pollution par les déchets semble être moins ressentie par les populations dans les quartiers résidentiels. A titre illustratif, les résultats, suivant ont été observés : pollution de l'air (35,66\%) ; pollution des sols (41,66\%) ; pollution des eaux (15,07\%). Pour les mêmes types de pollutions les résultats sont respectivement de $45,54 \%$; $42,41 \%$ et $53,95 \%$ (tableau 5) dans les quartiers populaires (bas standing). Ces différences se 
justifient par le fait que l'ensemble des quatre villes, les structures de pré collecte des déchets ne desservent que les quartiers résidentiels délaissant les quartiers dits «pauvres». Dans certains cas, les déchets sont collectés des quartiers de haut standing et déplacés vers les quartiers populaires (Babio, 2012), rendant encore l'environnement plus insalubre aux pauvres.

Tableau 5 : Perception des nuisances environnementales liées aux déchets ménagers selon le type de quartiers

\begin{tabular}{|c|c|c|c|c|c|c|c|c|}
\hline \multirow{3}{*}{\multicolumn{2}{|c|}{$\begin{array}{l}\text { Nuisances } \\
\text { environnementales }\end{array}$}} & \multicolumn{6}{|c|}{ Types de quartiers } & \multirow{3}{*}{ Total } \\
\hline & & \multicolumn{2}{|c|}{$\begin{array}{l}\text { Haut standing } \\
\text { (résidentiels) }\end{array}$} & \multicolumn{2}{|c|}{ Moyen standing } & \multicolumn{2}{|c|}{$\begin{array}{l}\text { Bas standing } \\
\text { (Quartiers populaires) }\end{array}$} & \\
\hline & & $\mathrm{N}$ & $\%$ & $\mathrm{~N}$ & $\%$ & $\mathrm{~N}$ & $\%$ & \\
\hline Pollution de l'air & & 12 & 32,43 & 104 & 37,68 & 97 & 35,66 & 213 \\
\hline Pollution des sols & & 14 & 37,84 & 115 & 41,66 & 95 & 34,93 & 224 \\
\hline Pollution des eaux & & 5 & 13,51 & 30 & 10,87 & 41 & 15,07 & 76 \\
\hline Dénaturation & de & & & & & & & \\
\hline $\begin{array}{l}\text { l'environnement } \\
\text { urbain }\end{array}$ & & 4 & 10,81 & 7 & 2,54 & 19 & 6,99 & 30 \\
\hline $\begin{array}{l}\text { Pollution } \\
\text { dénaturation } \\
\text { l'environnement }\end{array}$ & $\begin{array}{l}\text { et } \\
\text { de }\end{array}$ & 2 & 4,40 & 20 & 7,25 & 20 & 7,35 & 42 \\
\hline Total & & 37 & 100 & 276 & 100 & 272 & 100 & 585 \\
\hline
\end{tabular}

A travers ces résultats et leur analyse, il faut retenir que le degré de sensibilité des individus aux nuisances environnementales dues aux déchets solides ménagers est essentiellement lié au milieu de vie, donc de la proximité de la source de nuisance, au niveau d'instruction, à la catégorie socioprofessionnelle et dans une moindre mesure au revenu. La connaissance de ces déterminants est une donnée très précieuse pour l'aide à la prise de décisions dans les politiques de gestion écologique des déchets solides ménagers.

\section{Discussion}

Les résultats des recherches effectuées dans les villes de Parakou, Djougou, Kandi et Malanville ont révélé que la sensibilité aux nuisances environnementales liées aux DSM dépend de la ville de résidence. En effet, la pollution de l'eau, du sol et de l'air par les déchets ménagers sont des nuisances spécifiques à chaque agglomération. Elle dépend des mesures mises en place par les autorités locales pour la pré collecte des déchets dans les ménages et leur stockage dans des endroits appropriés. Ces résultats sont corroborés par les travaux de Thériault et al., (1995) à Port neuf au Canada qui ont souligné que la perception des risques environnementaux est liée au milieu géographique. Pour Des Rosiers et al. (1999) la sensibilité aux nuisances environnementales est fonction de la proximité du risque, donc du 
lieu de résidence. Du point de vue de l'âge, aucun lien statistique n’a été observé entre perception des nuisances environnementales et l'âge des individus interrogés. Samdahl et Robertson (1989) ont obtenu des résultats similaires en indiquant des relations négatives entre l'âge et la perception des nuisances environnementales. Par contre les travaux de Ostry et al., (1993) en Colombie britannique au Canada ont montré une sensibilité élevée des répondants jeunes mais mariés à la pollution de l'air par les déchets. Ces résultats sont similaires à ceux enregistrés dans les villes de Parakou, Djougou, Kandi et Malanville pour les 21 à 30 ans ; 31 à 40 ans et 41 à 50 ans. En effet, à ces différentes tranches d'âges, l'individu est très actif, se déplaçant beaucoup et donc plus exposé aux divers polluants atmosphériques. Par ailleurs, aucun lien statistique n’a été aussi obtenu entre le sexe des interrogés et les perceptions des nuisances environnementales. Il faut signaler toutefois une sensibilité relativement élevée des hommes à la pollution de l'air et ceci en raison de leur extrême mobilité. Du point de vue du niveau d'instruction, des liens statistiques significatifs ont été obtenu entre niveaux d'instruction et perception des nuisances environnementales liées aux déchets solides ménagers. En effet, avec un niveau d'instruction élevé, l'individu est mieux informé sur les problèmes d'hygiène et leurs conséquences sur l'environnement et la santé publique. C'est pourquoi, les répondants instruits sont plus sensibles aux nuisances environnementales liées aux déchets ménagers que les non instruits. Kientga, (2008) est parvenu aux conclusions similaires sur la perception du danger dû aux déchets ménagers à Ouagadougou. Les études portant sur la perception des nuisances environnementales réalisée par Theriault et al., (1995) sur les résidents de Portneuf au Canada est parvenue également aux résultats similaires. Mais Samdahl et Robertson (1989) ont trouvé une relation négative entre les questions environnementales et le degré d'instruction. De même les travaux de Bord et O'connor, (1992) ont montré qu'il n'y a pratiquement pas de liens entre le niveau d'éducation et les préoccupations environnementales. Ces différences peuvent s'expliquer par le fait que la présente étude s'est focalisée sur les risques environnementaux spécifiques aux déchets solides ménagers alors que Samdahl et Robertson (1989) ont analysé les préoccupations environnementales dans leur globalité. Quant aux travaux de Bord et O'connor, (1992), ils ont été réalisés sur un scénario fictif avec un échantillon réduit. Donc ces résultats doivent être pris avec une extrême prudence. Face au revenu individuel, la présente recherche n’a révélé aucun lien statistique significatif entre perception des nuisances environnementales et revenu des ménages. Par contre Kientga, (2008) et Neuman, (1986) ont souligné que les dangers aux risques environnementaux sont perçus à un degré élevé par les ménages à plus haut revenu. 


\section{Conclusion}

La perception des nuisances environnementales liées aux DSM permet de mesurer de l'acceptation de la présence des dépotoirs de déchets proche des habitations. Dans les villes de Parakou, Djougou, Kandi et Malanville au nord-Bénin, la perception des nuisances environnementales liées aux DSM dépend de la ville de résidence, du niveau d’instruction et de la catégorie socio-professionnelle des répondants. Le niveau de revenu, le sexe et l'âge des personnes interrogées dans l'ensemble des villes étudiées ne sont pas des indicateurs permettant d'établir des liens statistiques significatifs avec la perception sur les nuisances environnementales liées aux déchets solides ménagers. Toutefois, les femmes plus que les hommes, ont signalé la pollution des sols liée au DSM alors que les hommes s’attardent davantage à la pollution de l'air. De même, chez les répondants actifs : 21 à 30 ans ; 31 à 40 ans et 41 à 50 ans, le degré de sensibilité à la pollution de l'air est plus élevé que chez les personnes de 61 à plus de 70 ans. Au total, la proximité de la source de nuisance (spécificités géographiques de chaque lieu de résidence) et certains attributs individuels sociaux et parfois économiques déterminent la perception des nuisances liées aux DSM.

\section{References:}

Adeola F.O. 1994. Environmental hazards, health and racial iniquity in hazardous waste distribution.

Environ. Behav., 26 (1), 99-126

Babio S., 2012. Analyse de la vulnérabilité et des risques liés à l'exposition aux décharges sauvages de déchets solides ménagers dans les communes de Parakou et de N'dali au nord Bénin par l'utilisation du Système d’Information Géographique (SIG).

Mémoire pour l'obtention du Master 2 en Sciences de la Santé, de l'Environnement, du Territoire et de la Société. UNIVERSITE DE VERSAILLES SAINT-QUENTIN-EN-YVELINES (FRANCE), 80pg

Barnier J. 2013. Tout ce que vous n'avez jamais voulu savoir sur le $\chi 2$ sans jamais avoir eu envie de le demander.

Centre Max Weber. CNRS - UMR 5283, 38pg

Bord R. J. O'conncor R. E. 1992. Determinants of risk perceptions of a hazardous waste site.

Risk Anal., 12(3), 441-416

Bradbury, J. A., 1989. The policy implications of differing concepts of risk.

Science, Technology and Human Values, 14(4): 380-399

Canter L. W., Nelson D. I. and Everett J. W., 1994. Public perceptions of water quality risks - Influencing factors and enhancement opportunities.

Journal of Environmental Systems, 22(2): 163-187

Cvetkovich, G. and Earle T. C., 1992. Environmental hazards and the public. 
Journal of Social Issues, 48(4): 1-20.

Gbinlo R. E. 2010). Organisation et financement de la gestion des déchets ménagers dans les villes de l'Afrique Sub-saharienne : Cas de la ville de Cotonou au Bénin.

Thèse de Doctorat en Sciences Economiques Université d'Orléans ; 227 pg Guengant J-P. 2011. Comment bénéficier du dividende démographique ? La démographie au centre des trajectoires de développement dans les pays de l'UEMOA. Analyse pays Bénin, AFD, 55p.

Hamilton, L. C., 1985. Who cares about water pollution? Opinions in a small-town crisis. Sociological Inquiry, 55(2): 170-181

Kientga S., 2008. Contribution du SIG à l'analyse des liens déchets-santé en milieu urbain dans les pays en développement. Cas de deux secteurs de la ville de Ouagadougou, Burkina Faso.

Thèse pour l'obtention du grade de docteur ès sciences, École polytechnique fédérale de Lausanne, 213p

INSAE. 2015. RGPH4 : Que retenir des effectifs de population en 2013.

Direction des Etudes Démographiques, Cotonou, 35 pg.

Lehu J-M. 2004. La perception. L’encyclopédie du marketing.

Edition d'organisation, collection Références, parue le 02/09/2004, EAN13 : 9782708131453, 956 pages

Neuman, K. 1986. Personal values and commitment to energy conservation.

Environment and Behavior. 18: 53-74

Onibokun A. G. 2002. La Gestion des déchets urbains. Des solutions pour l'Afrique.

Editions Karthala, Paris CRDI, 260 p

Ostry A. S. Hertzman C. and Teschke K. 1993. Community risk perception : a case suty in a rural community hosting a waste site used by a large municipality.

Can. J.public health, 1993, 84, (6), 415-418

Raab M. et Gobin L. 2010. Les déchets à Nairobi.

Rapport d'expertise. Ingénierie des services urbains en réseau dans les pays en développent, Université de Renne, TELECOM Bretagne, ISTED, 56p

Renn, O., Burns W. J., Kasperson J. X., Kasperson R. E. and Slovic P. 1992. The social amplification of risk : Theoretical foundations and empirical applications.

Journal of Social Issues, 48(4): 137-160

Samdahl, D. M. and Robertson R. 1989. Social determinants of environmental concern : Specification and Test of the Model.

Environment and Behavior, 21: 57-81.

Theriault M., Landry R., Rouffignat J., Levallois P., Chiasson Ch. Tessier S. \& Girard M., Previl C. 1995. La perception des nuisances environnementales 
par les residents de Portneuf. Programme Eco-Recherche 922-94-00, Université de Laval Québec, Canada, 24pg

Des Rosiers, F. Bolduc A. and Thériault M. 1999. Environment and value: Does drinking water quality affect house prices?

Journal of Property Valuation and Investment \& finance, vol.17 I11; 5 pp.444-463.

Vining, J., Linn N. \& Burdge R. J., 1992. Why recycle? Acomparison of recycling motivations in four communities.

Environmental Management, 16(6): 785-797

Wandan E. N. ; Ngoran Ngoran J., Kouadio Kouakou B. \& Beda K. J. G. 2014. Perceptions des problèmes environnementaux dans la commune de Yamoussoukro en côte d'ivoire.

European Scientific Journal, vol.10, No.8 ISSN : 1857 - 7881 (Print) e ISSN 1857- 7431

Wang H., He J., Kim Y. \& Kamata T., 2014. Municipal solid waste management in rural areas and small countries: An economic analysis using contingent valuation to estimate willingness to pay for Yunnan, China.

Waste Manag Res. ; 32(8):695-706 